\title{
Highly Dispersive Optical Solitons with Complex Ginzburg-Landau Equation Having Six Nonlinear Forms
}

\author{
Elsayed M. E. Zayed ${ }^{1,+}{ }^{\text {, Khaled A. Gepreel }}{ }^{1,2,+} \mathbb{D}$, Mahmoud El-Horbaty ${ }^{1,+}$, Anjan Biswas ${ }^{3,4,5,6,7, *,+} \mathbb{D}$, \\ Yakup Yıldırım ${ }^{8,+}$ and Hashim M. Alshehri ${ }^{4,+}$
}

1 Mathematics Department, Faculty of Sciences, Zagazig University, Zagazig 44519, Egypt; eme_zayed@yahoo.com (E.M.E.Z.); k.gepreel@tu.edu.sa (K.A.G.); mahmoudelhorbaty8@gmail.com (M.E.-H.)

2 Mathematics Department, Faculty of Sciences, Taif University, Taif 21944, Saudi Arabia

3 Department of Applied Mathematics, National Research Nuclear University, 31 Kashirskoe Hwy, 115409 Moscow, Russia

4 Mathematical Modeling and Applied Computation (MMAC) Research Group, Department of Mathematics, King Abdulaziz University, Jeddah 21589, Saudi Arabia; hmalshehri@kau.edu.sa

5 Department of Applied Sciences, Cross-Border Faculty, Dunarea de Jos University of Galati, 111 Domneasca Street, 800201 Galati, Romania

6 Department of Mathematics and Applied Mathematics, Sefako Makgatho Health Sciences University, Ga-Rankuwa 0204, South Africa

7 Department of Physics, Chemistry and Mathematics, Alabama A\&M University, Huntsville, AL 35762 4900, USA

8 Department of Mathematics, Faculty of Arts and Sciences, Near East University, Nicosia 99138, Cyprus; yakupyildirim110@gmail.com

check for updates

Citation: Zayed, E.M.E.; Gepreel, K.A.; El-Horbaty, M.; Biswas, A.; Yıldırım, Y.; Alshehri, H.M. Highly Dispersive Optical Solitons with Complex Ginzburg-Landau Equation Having Six Nonlinear Forms. Mathematics 2021, 9, 3270. https:/ / doi.org/10.3390/math9243270

Academic Editor: Nikolai A. Kudryashov

Received: 18 November 2021 Accepted: 14 December 2021 Published: 16 December 2021

Publisher's Note: MDPI stays neutral with regard to jurisdictional claims in published maps and institutional affiliations.
* Correspondence: biswas.anjan@gmail.com

+ These authors contributed equally to this work.

\begin{abstract}
This paper retrieves highly dispersive optical solitons to complex Ginzburg-Landau equation having six forms of nonlinear refractive index structures for the very first time. The enhanced version of the Kudryashov approach is the adopted integration tool. Thus, bright and singular soliton solutions emerge from the scheme that are exhibited with their respective parameter constraints.
\end{abstract}

Keywords: solitons; refractive index; Kudryashov

\section{Introduction}

The physics and technology of optical solitons in telecommunications industry has totally revolutionized the modern world of quantum communications. The dynamics of soliton propagation through a variety of waveguides [1-3], as well as the modern study of meta-optics covers it all. Later, the concept of highly dispersive (HD) optical solitons [4-8] that was conceived during 2019 has theoretically addressed a growing problem in the modern telecommunications industry. This is the low count of chromatic dispersion (CD) that is a key element in sustaining the much needed balance between it and the self-phase modulation (SPM). HD solitons provide additional sources of dispersion to maintain this key balance between CD and SPM for the smooth propel of solitons through optical fibers for trans-continential and trans-oceanic distances. These additional sources of dispersion are from inter-modal dispersion (IMD), third-order dispersion (3OD), fourthorder dispersion (4OD), fifth-order dispersion (5OD), and sixth-order dispersion (6OD). These lead to the concept of HD solitons although, technically, dispersive effects would dominate the soliton propagation. Another shortcoming would be the drastic slow-down of solitons with such a collective dispersive count.

When HD solitons first came into existence, it was on the platform of nonlinear Schrödinger's equation (NLSE) [9-12]. After the concept of HD solitons was first reported, several works from this area have flooded a variety of journals over the last couple of years [13-17]. This, in fact, includes addressing of solitons with eighth-order dispersion. 
The current paper is addressing, for the first time, HD solitons on a different platform, namely the complex Ginzburg-Landau equation (CGLE) [18-26]. There are six forms of nonlinear refractive index structures that are considered. The integration scheme is the enhanced Kudryashov approach that reveals bright and singular optical solitons for each of these six nonlinear forms. These are exhibited and their respective parameter constraint conditions are also displayed. The detailed analysis are pen-pictured after a quick intro to the model.

\section{Governing Model}

The perturbed HD-CGLE that is considered for the very first time in this paper is indicated below

$$
\begin{aligned}
& i q_{t}+i a_{1} q_{x}+a_{2} q_{x x}+i a_{3} q_{x x x}+a_{4} q_{x x x x}+i a_{5} q_{x x x x x}+a_{6} q_{x x x x x x}+F\left(|q|^{2}\right) q \\
= & \alpha \frac{\left|q_{x}\right|^{2}}{q^{*}}+\frac{\beta}{4|q|^{2} q^{*}}\left\{2|q|^{2}\left(|q|^{2}\right)_{x x}-\left[\left(|q|^{2}\right)_{x}\right]^{2}\right\} \\
+ & \gamma q+i\left[\lambda\left(|q|^{2 m} q\right)_{x}+\mu\left(|q|^{2 m}\right)_{x} q+v|q|^{2 m} q_{x}\right],
\end{aligned}
$$

where $q=q(x, t)$ denotes the wave profile and $q^{*}$ represents the complex conjugate of the field $q=q(x, t)$, while $t$ and $x$ represents temporal and spatial variables, sequentially. $a_{j}$ $(j=1,2, \cdots, 6)$ are the coefficients of IMD, CD, 3OD, 4OD, 5OD, and 6OD. The first term is linear temporal evolution and $i=\sqrt{-1}$. $\gamma$ gives the detuning effect. $\lambda$ is the coefficient of self-steepening. $\mu$ is the coefficient of higher-order dispersion. $v$ is the coefficient of nonlinear dispersion. $\beta$ and $\alpha$ are the coefficients of nonlinear term. Lastly, $F\left(|q|^{2}\right)$ stands for nonlinear form.

Equation (1) is a generalized version of the perturbed CGLE [27-31]

$$
\begin{aligned}
& i q_{t}+i a q_{x x x}+F\left(|q|^{2}\right) q=\alpha \frac{\left|q_{x}\right|^{2}}{q^{*}}+\frac{\beta}{4|q|^{2} q^{*}}\left\{2|q|^{2}\left(|q|^{2}\right)_{x x}-\left[\left(|q|^{2}\right)_{x}\right]^{2}\right\} \\
& +\gamma q+i\left[\delta q_{x}+\lambda\left(|q|^{2 m} q\right)_{x}+\mu\left(|q|^{2 m}\right)_{x} q+v|q|^{2 m} q_{x}\right] .
\end{aligned}
$$

This paper studies the perturbed HD-CGLE (1) with six nonlinear forms using the integration methodology. The current paper is structured as: In Section 2, the perturbed HD-CGLE (1) is analyzed. In Section 3, the integration methodology is presented. In Sections 4-9, we arrive soliton solutions with the proposed models. The results of the paper are discussed in Section 10.

\section{Mathematical Preliminaries}

We presume the traveling wave transformation

$$
q(x, t)=\phi(\xi) e^{i\left(-\kappa x+w t+\theta_{0}\right)}, \xi=x-c t,
$$

where $\phi(\xi)$ is the amplitude of the traveling wave, $\kappa$ is the frequency, $c$ is the velocity, $w$ is the wave number and $\theta_{0}$ is the phase constant. Substituting (3) into (1) gives the real part

$$
\begin{aligned}
& a_{6} \phi \phi^{(6)}+\left(5 a_{5} \kappa+a_{4}-15 a_{6} \kappa^{2}\right) \phi \phi^{(4)} \\
+ & \left(15 a_{6} \kappa^{4}-10 a_{5} \kappa^{3}-6 a_{4} \kappa^{2}+3 a_{3} \kappa+a_{2}-\beta\right) \phi \phi^{\prime \prime} \\
- & \alpha\left(\phi^{\prime}\right)^{2}-\phi^{2}\left[a_{6} \kappa^{6}-a_{5} \kappa^{5}-a_{4} \kappa^{4}+a_{3} \kappa^{3}+\left(\alpha+a_{2}\right) \kappa^{2}-a_{1} \kappa+\gamma+w\right] \\
- & \kappa(v+\lambda) \phi^{2 m+2}+F\left(\phi^{2}\right) \phi^{2}=0,
\end{aligned}
$$


and the imaginary part

$$
\begin{gathered}
{\left[6 a_{6} \kappa-a_{5}\right] \phi^{(5)}+\left(6 a_{6} \kappa^{5}-4 a_{4} \kappa^{3}-5 a_{5} \kappa^{4}+2 a_{2} \kappa+3 a_{3} \kappa^{2}-a_{1}+c\right) \phi^{\prime}} \\
+\quad \phi^{2 m} \phi^{\prime}(2 m \lambda+2 m \mu+\lambda+v)+\phi^{\prime \prime \prime}\left(-20 a_{6} \kappa^{3}+10 a_{5} \kappa^{2}+4 a_{4} \kappa-a_{3}\right)=0 .
\end{gathered}
$$

Equation (5) yields the velocity

$$
c=-6 a_{6} \kappa^{5}+4 a_{4} \kappa^{3}+5 a_{5} \kappa^{4}-2 a_{2} \kappa-3 a_{3} \kappa^{2}+a_{1}
$$

and the frequency

$$
\kappa=\frac{a_{5}}{6 a_{6}}
$$

and the constraint conditions

$$
2 m \lambda+2 m \mu+\lambda+v=0,-20 a_{6} \kappa^{3}+10 a_{5} \kappa^{2}+4 a_{4} \kappa-a_{3}=0 .
$$

Equation (4) can be written as

$$
\phi \phi^{(6)}+\Delta_{1} \phi \phi^{(4)}+\Delta_{2} \phi \phi^{\prime \prime}+\Delta_{3}\left(\phi^{\prime}\right)^{2}+\Delta_{4} \phi^{2}+\Delta_{5} \phi^{2 m+2}+F\left(\phi^{2}\right) \phi^{2}=0,
$$

where

$$
\begin{aligned}
& \Delta_{1}=\frac{5 a_{5} \kappa+a_{4}-15 a_{6} \kappa^{2}}{a_{6}}, \Delta_{5}=-\frac{\kappa(v+\lambda)}{a_{6}}, \Delta_{3}=-\frac{\alpha}{a_{6}}, \\
& \Delta_{2}=\frac{15 a_{6} \kappa^{4}-10 a_{5} \kappa^{3}-6 a_{4} \kappa^{2}+3 a_{3} \kappa+a_{2}-\beta}{a_{6}}, \\
& \Delta_{4}=-\frac{a_{3} \kappa^{3}-a_{5} \kappa^{5}+a_{6} \kappa^{6}-a_{4} \kappa^{4}+\left(a_{2}+\alpha\right) \kappa^{2}+\gamma-a_{1} \kappa+w}{a_{6}} .
\end{aligned}
$$

\section{Enhanced Kudryashov Method}

The integration approach permits the formal solution

$$
\phi(\xi)=\sum_{g=0}^{N} \sigma_{g}[R(\xi)]^{g}, \sigma_{g} \neq 0,
$$

where $N$ is the order of the pole, $\sigma_{g}(g=0,1,2, \cdots, N)$ are arbitrary constants and $R(\xi)$ satisfies the ordinary differential equation

$$
R^{\prime 2}(\xi)=R^{2}(\xi)\left[1-\chi R^{2 p}(\xi)\right] \ln ^{2} K, 0<K \neq 1,
$$

along with the analytical solution

$$
R(\xi)=\left[\frac{4 A}{4 A^{2} \exp _{K}(p \xi)+\chi \exp _{K}(-p \xi)}\right]^{\frac{1}{p}} .
$$

Here $\chi, p$ and $A$ are non-zero real constants and $\exp _{K}(p \xi)=K^{p \xi}$. Plugging (11) along with (12) into (9) yields the coefficients $\sigma_{g}(g=0,1,2, \cdots, N)$. Substituting the coefficients $\sigma_{g}(g=0,1,2, \cdots, N)$ together with (13) into (11), we arrive the analytical solution of the model Equation (1).

\section{Kerr Law}

The Kerr law of nonlinearity is considered as

$$
F\left(\phi^{2}\right)=e \phi^{2}, e \neq 0,
$$


where $e$ is an arbitrary constant. Therefore, Equation (1) turns into

$$
\begin{aligned}
& i q_{t}+i a_{1} q_{x}+a_{2} q_{x x}+i a_{3} q_{x x x}+a_{4} q_{x x x x}+i a_{5} q_{x x x x x}+a_{6} q_{x x x x x x}+e|q|^{2} q \\
= & \alpha \frac{\left|q_{x}\right|^{2}}{q^{*}}+\frac{\beta}{4|q|^{2} q^{*}}\left\{2|q|^{2}\left(|q|^{2}\right)_{x x}-\left[\left(|q|^{2}\right)_{x}\right]^{2}\right\} \\
+ & \gamma q+i\left[\lambda\left(|q|^{2 m} q\right)_{x}+\mu\left(|q|^{2 m}\right)_{x} q+v|q|^{2 m} q_{x}\right],
\end{aligned}
$$

while Equation (9) simplifies to

$$
\phi \phi^{(6)}+\Delta_{1} \phi \phi^{(4)}+\Delta_{2} \phi \phi^{\prime \prime}+\Delta_{3}\left(\phi^{\prime}\right)^{2}+\Delta_{4} \phi^{2}+\Delta_{5} \phi^{2 m+2}+e \phi^{4}=0 .
$$

Setting $m=1$, Equation (16) collapses to

$$
\phi \phi^{(6)}+\Delta_{1} \phi \phi^{(4)}+\Delta_{2} \phi \phi^{\prime \prime}+\Delta_{3}\left(\phi^{\prime}\right)^{2}+\Delta_{4} \phi^{2}+\left(\Delta_{5}+e\right) \phi^{4}=0 .
$$

Balancing $\phi \phi^{(6)}$ and $\phi^{4}$ gives rise to

$$
2 N+6 p=4 N \Longrightarrow N=3 p .
$$

Case 1: With the help of $p=1$, Equation (11) turns into

$$
\phi(\xi)=\sigma_{0}+\sigma_{1} R(\xi)+\sigma_{2} R^{2}(\xi)+\sigma_{3} R^{3}(\xi), \sigma_{3} \neq 0,
$$

where $\sigma_{0}, \sigma_{1}, \sigma_{2}$ and $\sigma_{3}$ are arbitrary constants. Substituting (19) along with (12) into (17) causes the coefficients to

$$
\sigma_{0}=\sigma_{1}=\sigma_{2}=0, \sigma_{3}=24 \varepsilon \chi \sqrt{\frac{35 \chi}{\Delta_{5}+e}} \ln ^{3} K,
$$

and the constraint conditions

$$
\begin{aligned}
& \Delta_{1}=-83 \ln ^{2} K, \Delta_{3}=-\frac{\Delta_{2}-7564 \ln ^{4} K}{3} \\
& \Delta_{4}=\left(3 \Delta_{2}-16698 \ln ^{4} K\right) \ln ^{2} K, \chi\left(\Delta_{5}+e\right)>0, \varepsilon= \pm 1 .
\end{aligned}
$$

Plugging (20) together with (13) and (21) into (19), the straddled soliton is formulated as

$$
\begin{aligned}
q(x, t)= & 24 \varepsilon \chi\left(\ln ^{3} K\right) \sqrt{\frac{35 \chi}{\Delta_{5}+e}}\left[\frac{4 A}{4 A^{2} \exp _{K}(x-c t)+\chi \exp _{K}(-(x-c t))}\right]^{3} \\
& \times e^{i\left(-\kappa x+w t+\theta_{0}\right)} .
\end{aligned}
$$

By the aid of $\Delta_{5}+e>0$ and $\chi=4 A^{2}$, the bright soliton is indicated below

$$
q(x, t)=24 \varepsilon\left(\ln ^{3} K\right) \sqrt{\frac{35}{\Delta_{5}+e}} \operatorname{sech}^{3}[(x-c t) \ln K] e^{i\left(-\kappa x+w t+\theta_{0}\right)} .
$$

By the usage of $\Delta_{5}+e<0$ and $\chi=-4 A^{2}$, the singular soliton is considered as

$$
q(x, t)=24 \varepsilon\left(\ln ^{3} K\right) \sqrt{-\frac{35}{\Delta_{5}+e}} \operatorname{csch}^{3}[(x-c t) \ln K] e^{i\left(-\kappa x+w t+\theta_{0}\right)} .
$$


Case 2: With the help of $p=2$, Equation (11) transforms to

$$
\phi(\xi)=\sigma_{0}+\sigma_{1} R(\xi)+\sigma_{2} R^{2}(\xi)+\sigma_{3} R^{3}(\xi)+\sigma_{4} R^{4}(\xi)+\sigma_{5} R^{5}(\xi)+\sigma_{6} R^{6}(\xi), \sigma_{6} \neq 0,
$$

where $\sigma_{i}(i=0,1, \cdots, 6)$ are arbitrary constants and $R(\xi)$ changes to

$$
R^{\prime 2}(\xi)=R^{2}(\xi)\left[1-\chi R^{4}(\xi)\right] \ln ^{2} K, 0<K, K \neq 1 .
$$

Inserting (25) along with (26) into (17) yields the coefficients

$$
\sigma_{0}=\sigma_{1}=\sigma_{2}=\sigma_{3}=\sigma_{4}=\sigma_{5}=0, \sigma_{6}=-192 \varepsilon \chi \sqrt{\frac{35 \chi}{\Delta_{4}+e}} \ln ^{3} K,
$$

and the parameter constraints

$$
\begin{aligned}
& \Delta_{1}=-332 \ln ^{2} K, \Delta_{3}=-\frac{4 \Delta_{2}-121024 \ln ^{4} K}{3} \\
& \Delta_{4}=\left(12 \Delta_{2}-1068672 \ln ^{4} K\right) \ln ^{2} K, \chi\left(\Delta_{5}+e\right)>0, \varepsilon= \pm 1 .
\end{aligned}
$$

Putting (27) together with (13) and (28) into (25), the straddled soliton is structured as

$$
\begin{aligned}
q(x, t)= & -192 \varepsilon \chi\left(\ln ^{3} K\right) \sqrt{\frac{35 \chi}{\Delta_{4}+e}}\left[\frac{4 A}{4 A^{2} \exp _{K}[2(x-c t)]+\chi \exp _{K}[2(x-c t)]}\right]^{3} \\
& \times e^{i\left(-\kappa x+w t+\theta_{0}\right)} .
\end{aligned}
$$

By the aid of $\Delta_{5}+e>0$ and $\chi=4 A^{2}$, the bright soliton is formulated as

$$
q(x, t)=-192 \varepsilon\left(\ln ^{3} K\right) \sqrt{\frac{35}{\Delta_{5}+e}} \operatorname{sech}^{3}[2(x-c t) \ln K] e^{i\left(-\kappa x+w t+\theta_{0}\right)} .
$$

By virtue of $\Delta_{5}+e<0$ and $\chi=-4 A^{2}$, the singular soliton is indicated below

$$
q(x, t)=192 \varepsilon\left(\ln ^{3} K\right) \sqrt{-\frac{35}{\Delta_{5}+e}} \operatorname{csch}^{3}[2(x-c t) \ln K] e^{i\left(-\kappa x+w t+\theta_{0}\right)} .
$$

\section{Power Law}

The power law of nonlinearity is structured as

$$
F\left(\phi^{2}\right)=e \phi^{2 n}, e \neq 0,
$$

where $e$ is an arbitrary constant. Thus, Equation (1) simplifies to

$$
\begin{aligned}
& i q_{t}+i a_{1} q_{x}+a_{2} q_{x x}+i a_{3} q_{x x x}+a_{4} q_{x x x x}+i a_{5} q_{x x x x x}+a_{6} q_{x x x x x x}+e|q|^{2 n} q \\
= & \alpha \frac{\left|q_{x}\right|^{2}}{q^{*}}+\frac{\beta}{4|q|^{2} q^{*}}\left\{2|q|^{2}\left(|q|^{2}\right)_{x x}-\left[\left(|q|^{2}\right)_{x}\right]^{2}\right\} \\
+ & \gamma q+i\left[\lambda\left(|q|^{2 m} q\right)_{x}+\mu\left(|q|^{2 m}\right)_{x} q+v|q|^{2 m} q_{x}\right],
\end{aligned}
$$

while Equation (9) collapses to

$$
\phi \phi^{(6)}+\Delta_{1} \phi \phi^{(4)}+\Delta_{2} \phi \phi^{\prime \prime}+\Delta_{3}\left(\phi^{\prime}\right)^{2}+\Delta_{4} \phi^{2}+\Delta_{5} \phi^{2 m+2}+e \phi^{2 n+2}=0 .
$$


Setting $n=m$, Equation (34) transforms to

$$
\phi \phi^{(6)}+\Delta_{1} \phi \phi^{(4)}+\Delta_{2} \phi \phi^{\prime \prime}+\Delta_{3}\left(\phi^{\prime}\right)^{2}+\Delta_{4} \phi^{2}+\left(\Delta_{5}+e\right) \phi^{2 m+2}=0 .
$$

Balancing $\phi \phi^{(6)}$ and $\phi^{2 m+2}$ yields $N=\frac{3}{m}$. Setting

$$
\phi=[U(\xi)]^{\frac{3}{m}},
$$

Equation (35) turns into

$$
\begin{aligned}
& -9(m-1)(2 m-3)(4 m-3)(5 m-3)(m-3) U^{\prime 6} \\
+ & 135 m(m-1)(2 m-3)(4 m-3)(m-3) U U^{\prime \prime} U^{\prime 4} \\
- & 9 \Delta_{1} m^{2}(m-1)(2 m-3)(m-3) U^{2} U^{\prime 4} \\
- & 180 m^{2}(m-1)(2 m-3)(m-3) U^{2} U^{\prime 3} U^{\prime \prime \prime}+\Delta_{4} m^{6} U^{6} \\
- & 405 m^{2}(m-1)(2 m-3)(m-3) U^{2} U^{\prime \prime 2} U^{\prime 2}+18 \Delta_{1} m^{3}(2 m-3)(m-3) U^{3} U^{\prime \prime} U^{\prime 2} \\
+ & 3 m^{5} U^{5} U^{(6)}+45 m^{3}(2 m-3)(m-3) U^{3} U^{(4)} U^{\prime^{2}}+3 m^{4}\left[3 \Delta_{3}-(m-3) \Delta_{2}\right] U^{4} U^{\prime 2} \\
- & 12 \Delta_{1} m^{4}(m-3) U^{4} U^{\prime \prime \prime} U^{\prime}+180 m^{3}(2 m-3)(m-3) U^{3} U^{\prime \prime \prime} U^{\prime \prime} U^{\prime} \\
- & 18 m^{4}(m-3) U^{4} U^{(5)} U^{\prime}+45 m^{3}(2 m-3)(m-3) U^{3}\left(U^{\prime \prime \prime}\right)^{3} \\
- & 9 \Delta_{1} m^{4}(m-3) U^{4}\left(U^{\prime \prime}\right)^{2}-45 m^{4}(m-3) U^{4} U^{(4)} U^{\prime \prime}+3 m^{5} \Delta_{2} U^{5} U^{\prime \prime} \\
+ & m^{6}\left(\Delta_{5}+e\right) U^{12}-30 m^{4}(m-3) U^{4}\left(U^{\prime \prime \prime}\right)^{2}+3 m^{5} \Delta_{1} U^{5} U^{(4)}=0 .
\end{aligned}
$$

Balancing $U^{5} U^{(6)}$ and $U^{12}$ leads to

$$
6 N+6 p=12 N \Longrightarrow N=p .
$$

Case 1: By virtue of $p=1$, Equation (11) collapses to

$$
U(\xi)=\sigma_{0}+\sigma_{1} R(\xi), \sigma_{1} \neq 0,
$$

where $\sigma_{0}$ and $\sigma_{1}$ are arbitrary constants. Plugging (39) along with (12) into (37), we arrive the coefficients

$$
\sigma_{0}=0, \sigma_{1}=\frac{\epsilon \ln K}{m}\left(\frac{9(4 m+3)(5 m+3)(2 m+3)(m+3)(m+1) \chi^{3}}{\Delta_{5}+e}\right)^{\frac{1}{6}},
$$

and the parameter constraints

$$
\begin{aligned}
& \Delta_{1}=-\frac{\left(20 m^{2}+36 m+27\right) \ln ^{2} K}{m^{2}}, \chi^{3}\left(\Delta_{5}+e\right)>0, \\
& \Delta_{3}=-\frac{\left(\begin{array}{c}
64 m^{5}+480 m^{4}+1512 m^{3} \\
+2592 m^{2}+2187 m+729
\end{array}\right) \ln ^{4} K}{3 m^{4}}-\frac{(m+3) \Delta_{2}}{3}, \\
& \Delta_{4}=-\frac{3\left(\begin{array}{c}
64 m^{5}+480 m^{4}+1512 m^{3} \\
+2052 m^{2}+1215 m+243
\end{array}\right) \ln ^{6} K}{m^{6}}+\frac{3 \Delta_{2} \ln ^{2} K}{m} .
\end{aligned}
$$


Inserting (40) together with (13) and (41) into (39), the straddled soliton is modeled as

$$
\begin{aligned}
q(x, t)= & \left\{\frac{4 \epsilon A\left(\frac{9(4 m+3)(2 m+3)(5 m+3)(m+1)(m+3) \chi^{3}}{\Delta_{5}+e}\right)^{\frac{1}{6}} \ln K}{m\left[4 A^{2} \exp _{K}(x-c t)+\chi \exp _{K}(-(x-c t))\right]}\right\}^{\frac{3}{m}} \\
& \times e^{i\left(-\kappa x+w t+\theta_{0}\right)} .
\end{aligned}
$$

By the usage of $\Delta_{5}+e>0$ and $\chi=4 A^{2}$, the bright soliton is structured as

$$
\left.\begin{array}{rl}
q(x, t)= & \left\{\frac{4 \epsilon \ln K}{m}\left(\frac{9(4 m+3)(2 m+3)(5 m+3)(m+1)(m+3)}{\Delta_{5}+e}\right)^{\frac{1}{6}}\right. \\
\times \operatorname{sech}[(x-c t) \ln K]
\end{array}\right\}
$$

With the help of $\Delta_{5}+e<0$ and $\chi=-4 A^{2}$, the singular soliton is indicated below

$$
\begin{aligned}
q(x, t)= & \left\{\frac{4 \epsilon \ln K}{m}\left(-\frac{9(4 m+3)(2 m+3)(5 m+3)(m+1)(m+3)}{\Delta_{5}+e}\right)^{\frac{1}{6}}\right. \\
\times \operatorname{csch}[(x-c t) \ln K] & \times e^{i\left(-\kappa x+w t+\theta_{0}\right)} .
\end{aligned}
$$

Case 2: By virtue of $p=2$, Equation (11) becomes

$$
U(\xi)=\sigma_{0}+\sigma_{1} R(\xi)+\sigma_{2} R^{2}(\xi), \sigma_{2} \neq 0,
$$

where $\sigma_{0}, \sigma_{1}$ and $\sigma_{2}$ are arbitrary constants. Putting (45) along with (12) into (37) causes to the coefficients

$$
\sigma_{0}=\sigma_{1}=0, \sigma_{2}=\frac{2 \epsilon \ln K}{m}\left(\frac{9(4 m+3)(2 m+3)(5 m+3)(m+1)(m+3) \chi^{3}}{\Delta_{5}+e}\right)^{\frac{1}{6}},
$$

and the constraints

$$
\begin{aligned}
& \Delta_{1}=-\frac{4\left(20 m^{2}+36 m+27\right) \ln ^{2} K}{m^{2}}, \chi^{3}\left(\Delta_{5}+e\right)>0, \\
& \Delta_{3}=\frac{16\left(\begin{array}{c}
64 m^{5}+480 m^{4}+1512 m^{3} \\
+2592 m^{2}+2187 m+729
\end{array}\right) \ln ^{4} K}{3 m^{4}}-\frac{(m+3) \Delta_{2}}{3}, \\
& \Delta_{4}=-\frac{192\left(\begin{array}{c}
64 m^{5}+480 m^{4}+1512 m^{3} \\
+2052 m^{2}+1215 m+243
\end{array}\right) \ln ^{6} K}{m^{6}}+\frac{12 \Delta_{2} \ln ^{2} K}{m} .
\end{aligned}
$$

Plugging (46) together with (13) and (47) into (45), the straddled soliton is considered as

$$
\begin{aligned}
q(x, t)= & \left\{\frac{8 \epsilon A \ln K\left(\frac{9(4 m+3)(2 m+3)(5 m+3)(m+1)(m+3) \chi^{3}}{e+\Delta_{5}}\right)^{\frac{1}{6}}}{m\left[4 A^{2} \exp _{K}[2(x-c t)]+\chi \exp _{K}[-2(x-c t)]\right]}\right\}^{\frac{3}{m}} \\
& \times e^{i\left(-\kappa x+w t+\theta_{0}\right)} .
\end{aligned}
$$


By the usage of $\Delta_{5}+e>0$ and $\chi=4 A^{2}$, the bright soliton is modeled as

$$
\begin{aligned}
q(x, t)= & \left\{\begin{array}{c}
\frac{2 \epsilon \ln K}{m}\left(\frac{9(4 m+3)(5 m+3)(2 m+3)(m+3)(m+1)}{e+\Delta_{5}}\right)^{\frac{1}{6}} \\
\times \operatorname{sech}[2 \ln K(x-c t)]
\end{array}\right\}^{\frac{3}{m}} \\
& \times e^{i\left(-\kappa x+w t+\theta_{0}\right)} .
\end{aligned}
$$

With the help of $\Delta_{5}+e<0$ and $\chi=-4 A^{2}$, the singular soliton is formulated as

$$
\left.\begin{array}{rl}
q(x, t)= & \left\{\frac{2 \epsilon \ln K}{m}\left(-\frac{9(4 m+3)(5 m+3)(2 m+3)(m+3)(m+1)}{e+\Delta_{5}}\right)^{\frac{1}{6}}\right. \\
\times \operatorname{csch}[2 \ln K(x-c t)]
\end{array}\right\}^{\frac{3}{m}}
$$

\section{Parabolic Law}

The parabolic law of nonlinearity is indicated below

$$
F\left(\phi^{2}\right)=e_{1} \phi^{2}+e_{2} \phi^{4}, e_{2} \neq 0,
$$

where $e_{1}$ and $e_{2}$ are arbitrary constants. Consequently, Equation (1) turns into

$$
\begin{aligned}
& i q_{t}+i a_{1} q_{x}+a_{2} q_{x x}+i a_{3} q_{x x x}+a_{4} q_{x x x x}+i a_{5} q_{x x x x x}+a_{6} q_{x x x x x x} \\
+ & \left(e_{1}|q|^{2}+e_{2}|q|^{4}\right) q=\alpha \frac{\left|q_{x}\right|^{2}}{q^{*}}+\frac{\beta}{4|q|^{2} q^{*}}\left\{2|q|^{2}\left(|q|^{2}\right)_{x x}-\left[\left(|q|^{2}\right)_{x}\right]^{2}\right\} \\
+ & \gamma q+i\left[\lambda\left(|q|^{2 m} q\right)_{x}+\mu\left(|q|^{2 m}\right)_{x} q+v|q|^{2 m} q_{x}\right],
\end{aligned}
$$

while Equation (9) decreases to

$$
\phi \phi^{(6)}+\Delta_{1} \phi \phi^{(4)}+\Delta_{2} \phi \phi^{\prime \prime}+\Delta_{3}\left(\phi^{\prime}\right)^{2}+\Delta_{4} \phi^{2}+\Delta_{5} \phi^{2 m+2}+e_{1} \phi^{4}+e_{2} \phi^{6}=0 .
$$

Setting $m=1$, Equation (53) becomes

$$
\phi \phi^{(6)}+\Delta_{1} \phi \phi^{(4)}+\Delta_{2} \phi \phi^{\prime \prime}+\Delta_{3}\left(\phi^{\prime}\right)^{2}+\Delta_{4} \phi^{2}+\left(\Delta_{5}+e_{1}\right) \phi^{4}+e_{2} \phi^{6}=0 .
$$

Balancing $\phi \phi^{(6)}$ and $\phi^{6}$ causes to $N=\frac{3}{2}$. Setting

$$
\phi(\xi)=[U(\xi)]^{\frac{3}{2}},
$$

Equation (54) collapses to

$$
\begin{aligned}
& 315\left[U^{\prime}\right]^{6}+36 \Delta_{1} U^{2} U^{\prime 4}-1350 U U^{\prime \prime} U^{\prime 4}+720 U^{2} U^{\prime 3} U^{\prime \prime \prime}+1620 U^{2} U^{\prime \prime 2} U^{\prime 2} \\
- & 144 \Delta_{1} U^{3} U^{\prime \prime} U^{\prime 2}-360 U^{3} U^{(4)} U^{\prime{ }^{2}}+48\left[3 \Delta_{3}+\Delta_{2}\right] U^{4} U^{\prime{ }^{2}}+192 \Delta_{1} U^{4} U^{\prime \prime \prime} U^{\prime} \\
+ & 288 U^{4} U^{(5)} U^{\prime}-1440 U^{3} U^{\prime \prime \prime} U^{\prime \prime} U^{\prime}-360 U^{3}\left(U^{\prime \prime}\right)^{3}+144 \Delta_{1} U^{4}\left(U^{\prime \prime}\right)^{2} \\
+ & 96 \Delta_{2} U^{5} U^{\prime \prime}+720 U^{4} U^{(4)} U^{\prime \prime}+480 U^{4}\left(U^{\prime \prime \prime}\right)^{2}+96 \Delta_{1} U^{5} U^{(4)}+96 U^{5} U^{(6)} \\
+ & 64 e_{2} U^{12}+64\left(e_{1}+\Delta_{5}\right) U^{9}+64 \Delta_{4} U^{6}=0 .
\end{aligned}
$$

Balancing $U^{5} U^{(6)}$ and $U^{12}$ gives rise to

$$
6 N+6 p=12 N \Longrightarrow N=p .
$$


Case 1: By the usage of $p=1$, Equation (56) permits the solution (39). Substituting (39) along with (12) into (56) gives rise to the coefficients

$$
\sigma_{0}=0, \sigma_{1}=\left(\frac{135135 \chi^{3}}{64 e_{2}}\right)^{\frac{1}{6}} \ln K,
$$

and the parameters

$$
\begin{aligned}
& \Delta_{1}=-\frac{179 \ln ^{2} K}{4}, \Delta_{3}=\frac{37295 \ln ^{4} K}{48}-\frac{5 \Delta_{2}}{3} \\
& \Delta_{4}=-\frac{98115 \ln ^{6} K}{64}+\frac{3 \Delta_{2} \ln ^{2} K}{2}, \Delta_{5}=-e_{1}, \chi^{3} e_{2}>0 .
\end{aligned}
$$

Plugging (58) together with (13) and (59) into (39), we arrive the straddled soliton

$$
q(x, t)=\left\{\frac{4 A\left(\frac{135135 \chi^{3}}{64 e_{2}}\right)^{\frac{1}{6}} \ln K}{4 A^{2} \exp _{K}(x-c t)+\chi \exp _{K}(-(x-c t))}\right\}^{i\left(-\kappa x+w t+\theta_{0}\right)} .
$$

Setting $e_{2}>0$ and $\chi=4 A^{2}$, Equation (60) transforms to the bright soliton

$$
q(x, t)=\left\{(\ln K)\left(\frac{135135}{64 e_{2}}\right)^{\frac{1}{6}} \operatorname{sech}[(x-c t) \ln K]\right\}^{\frac{3}{2}} e^{i\left(-\kappa x+w t+\theta_{0}\right)} .
$$

If $e_{2}<0$ and $\chi=-4 A^{2}$, Equation (60) yields the singular soliton

$$
q(x, t)=\left\{(\ln K)\left(-\frac{135135}{64 e_{2}}\right)^{\frac{1}{6}} \operatorname{csch}[(x-c t) \ln K]\right\}^{\frac{3}{2}} e^{i\left(-\kappa x+w t+\theta_{0}\right)} .
$$

Case 2: By the aid of $p=2$, Equation (56) holds the solution (45). Substituting (45) along with (12) into (56) leads to the coefficients

$$
\sigma_{0}=\sigma_{1}=0, \sigma_{2}=\left(\frac{135135 \chi^{3}}{e_{2}}\right)^{\frac{1}{6}} \ln K,
$$

and the constraints

$$
\begin{aligned}
& \Delta_{1}=-179 \ln ^{2} K, \Delta_{3}=\frac{37295 \ln ^{4} K}{3}-\frac{5 \Delta_{2}}{3} \\
& \Delta_{4}=\left(6 \Delta_{2}-98115 \ln ^{4} K\right) \ln ^{2} K, \Delta_{5}=-e_{1}, \chi^{3} e_{2}>0 .
\end{aligned}
$$

Inserting (63) together with (13) and (64) into (45), the straddled soliton is formulated as

$$
q(x, t)=\left\{\frac{4 A\left(\frac{135135 \chi^{3}}{e_{2}}\right)^{\frac{1}{6}} \ln K}{4 A^{2} \exp _{K}[2(x-c t)]+\chi \exp _{K}[-2(x-c t)]}\right\}^{\frac{3}{2}} e^{i\left(-\kappa x+w t+\theta_{0}\right)}
$$


If $e_{2}>0$ and $\chi=4 A^{2}$, Equation (65) becomes the bright soliton

$$
q(x, t)=\left\{(\ln K)\left(\frac{135135}{e_{2}}\right)^{\frac{1}{6}} \operatorname{sech}[2 \ln K(x-c t)]\right\}^{\frac{3}{2}} e^{i\left(-\kappa x+w t+\theta_{0}\right)} .
$$

When $e_{2}<0$ and $\chi=-4 A^{2}$, Equation (65) turns into the singular soliton

$$
q(x, t)=\left\{(\ln K)\left(-\frac{135135}{e_{2}}\right)^{\frac{1}{6}} \operatorname{csch}[2 \ln K(x-c t)]\right\}^{\frac{3}{2}} e^{i\left(-\kappa x+w t+\theta_{0}\right)} .
$$

\section{Dual Power Law}

The dual power law of nonlinearity is considered as

$$
F\left(\phi^{2}\right)=e_{1} \phi^{2 n}+e_{2} \phi^{4 n}, e_{2} \neq 0,
$$

where $e_{1}$ and $e_{2}$ are arbitrary constants. Hence, Equation (1) simplifies to

$$
\begin{aligned}
& i q_{t}+i a_{1} q_{x}+a_{2} q_{x x}+i a_{3} q_{x x x}+a_{4} q_{x x x x}+i a_{5} q_{x x x x x}+a_{6} q_{x x x x x x} \\
+ & \left(e_{1}|q|^{2 n}+e_{2}|q|^{4 n}\right) q=\alpha \frac{\left|q_{x}\right|^{2}}{q^{*}}+\frac{\beta}{4|q|^{2} q^{*}}\left\{2|q|^{2}\left(|q|^{2}\right)_{x x}-\left[\left(|q|^{2}\right)_{x}\right]^{2}\right\} \\
+ & \gamma q+i\left[\lambda\left(|q|^{2 m} q\right)_{x}+\mu\left(|q|^{2 m}\right)_{x} q+v|q|^{2 m} q_{x}\right],
\end{aligned}
$$

while Equation (9) collapses to

$$
\phi \phi^{(6)}+\Delta_{1} \phi \phi^{(4)}+\Delta_{2} \phi \phi^{\prime \prime}+\Delta_{3}\left(\phi^{\prime}\right)^{2}+\Delta_{4} \phi^{2}+\Delta_{5} \phi^{2 m+2}+e_{1} \phi^{2 n+2}+e_{2} \phi^{4 n+2}=0 .
$$

Setting $n=m$, Equation (70) decreases to

$$
\phi \phi^{(6)}+\Delta_{1} \phi \phi^{(4)}+\Delta_{2} \phi \phi^{\prime \prime}+\Delta_{3}\left(\phi^{\prime}\right)^{2}+\Delta_{4} \phi^{2}+\left(\Delta_{5}+e_{1}\right) \phi^{2 m+2}+e_{2} \phi^{4 m+2}=0 .
$$

Balancing $\phi \phi^{(6)}$ and $\phi^{4 m+2}$ gives $N=\frac{3}{2 m}$. Setting

$$
\phi(\xi)=[U(\xi)]^{\frac{3}{2 m}},
$$


Equation (71) turns into

$$
\begin{aligned}
& -9(2 m-3)(2 m-1)(4 m-3)(10 m-3)\left[U^{\prime}\right]^{6} \\
+ & 270 m(2 m-3)(2 m-1)(8 m-3)(4 m-3) U U^{\prime \prime} U^{\prime 4} \\
- & 36 m^{2}(2 m-3)(4 m-3)(2 m-1) \Delta_{1} U^{2} U^{\prime 4} \\
- & 720 m^{2}(2 m-3)(2 m-1)(4 m-3) U^{2} U^{\prime 3} U^{\prime \prime \prime} \\
- & 1620 m^{2}(2 m-1)(2 m-3)(4 m-3) U^{2} U^{\prime \prime 2} U^{\prime 2} \\
+ & 144 \Delta_{1} m^{3}(2 m-3)(4 m-3) U^{3} U^{\prime \prime} U^{\prime 2} \\
+ & 360 m^{3}(2 m-3)(4 m-3) U^{3} U^{(4)} U^{\prime 2}-48 m^{4}\left[(2 m-3) \Delta_{2}-3 \Delta_{3}\right] U^{4} U^{\prime 2} \\
+ & 1440 m^{3}(2 m-3)(4 m-3) U^{3} U^{\prime \prime \prime} U^{\prime \prime} U^{\prime}-192 \Delta_{1} m^{4}(2 m-3) U^{4} U^{\prime \prime \prime} U^{\prime} \\
- & 288 m^{4}(2 m-3) U^{4} U^{(5)} U^{\prime}+360 m^{3}(2 m-3)(4 m-3) U^{3}\left(U^{\prime \prime}\right)^{3} \\
- & 144 \Delta_{1} m^{4}(2 m-3) U^{4}\left(U^{\prime \prime}\right)^{2}-720 m^{4}(2 m-3) U^{4} U^{(4)} U^{\prime \prime} \\
+ & 96 m^{5} \Delta_{2} U^{5} U^{\prime \prime}-480 m^{4}(2 m-3) U^{4}\left(U^{\prime \prime \prime}\right)^{2}+96 m^{5} \Delta_{1} U^{5} U^{(4)} \\
+ & 96 m^{5} U^{5} U^{(6)}+64 m^{6} e_{2} U^{12}+64 m^{6}\left(e_{1}+\Delta_{5}\right) U^{9}+64 m^{6} \Delta_{4} U^{6}=0 .
\end{aligned}
$$

Balancing $U^{5} U^{(6)}$ and $U^{12}$ leads to

$$
6 N+6 p=12 N \Longrightarrow N=p
$$

Case 1: By the usage of $p=1$, Equation (73) satisfies the solution (39). Putting (39) along with (12) into (73), we arrive the coefficients

$$
\sigma_{0}=0, \sigma_{1}=\frac{\ln K}{m}\left(\frac{9 \chi^{3}(8 m+3)(4 m+3)(10 m+3)(2 m+3)(2 m+1)}{64 e_{2}}\right)^{\frac{1}{6}}
$$

and the constraints

$$
\begin{aligned}
& \Delta_{1}=-\frac{\left(80 m^{2}+72 m+27\right) \ln ^{2} K}{4 m^{2}}, \Delta_{5}=-e_{1}, \chi^{3} e_{2}>0 \\
& \Delta_{3}=\frac{\left(\begin{array}{c}
2048 m^{5}+7680 m^{4}+12096 m^{3} \\
+10368 m^{2}+4374 m+729
\end{array}\right) \ln ^{4} K}{48 m^{4}}-\frac{(2 m+3) \Delta_{2}}{3}, \\
& \Delta_{4}=-\frac{3\left(\begin{array}{c}
2048 m^{5}+7680 m^{4}+12096 m^{3} \\
+8208 m^{2}+2430 m+243
\end{array}\right) \ln ^{6} K}{64 m^{6}}+\frac{3 \Delta_{2}}{2 m} \ln ^{2} K .
\end{aligned}
$$

Inserting (75) together with (13) and (76) into (39), we arrive the straddled soliton

$$
\begin{aligned}
q(x, t)= & \left\{\frac{4 A\left(\frac{9 \chi^{3}(8 m+3)(4 m+3)(10 m+3)(2 m+3)(2 m+1)}{64 e_{2}}\right)^{\frac{1}{6}} \ln K}{m\left[4 A^{2} \exp _{K}(x-c t)+\chi \exp _{K}(-(x-c t))\right]}\right\}^{\frac{3}{2 m}} \\
& \times e^{i\left(-\kappa x+w t+\theta_{0}\right)} .
\end{aligned}
$$


By virtue of $e_{2}>0$ and $\chi=4 A^{2}$, Equation (77) simplifies to the bright soliton

$$
\begin{aligned}
q(x, t)= & \left\{\begin{array}{c}
\frac{\ln K}{m}\left(\frac{9(8 m+3)(4 m+3)(10 m+3)(2 m+3)(2 m+1)}{64 e_{2}}\right)^{\frac{1}{6}} \\
\times \operatorname{sech}[(x-c t) \ln K]
\end{array}\right\}^{\frac{3}{2 m}} \\
& \times e^{i\left(-\kappa x+w t+\theta_{0}\right)} .
\end{aligned}
$$

By the aid of $e_{2}<0$ and $\chi=-4 A^{2}$, Equation (77) collapses to the singular soliton

$$
\begin{aligned}
q(x, t)= & \left\{\frac{\ln K}{m}\left(-\frac{9(8 m+3)(4 m+3)(10 m+3)(2 m+3)(2 m+1)}{64 e_{2}}\right)^{\frac{1}{6}}\right. \\
& \times e^{i\left(-\kappa x+w t+\theta_{0}\right)} .
\end{aligned}
$$

Case 2: With the help of $p=2$, Equation (73) presumes the solution (45). Plugging (45) along with (12) into (73) yields the coefficients

$$
\sigma_{0}=\sigma_{1}=0, \sigma_{2}=\frac{\ln K}{m}\left(\frac{9 \chi^{3}(8 m+3)(4 m+3)(10 m+3)(2 m+3)(2 m+1)}{e_{2}}\right)^{\frac{1}{6}},
$$

and the conditions

$$
\begin{aligned}
& \Delta_{1}=-\frac{\left(80 m^{2}+72 m+27\right) \ln ^{2} K}{m^{2}}, \Delta_{5}=-e_{1}, \chi^{3} e_{2}>0, \\
& \Delta_{3}=\frac{\left(\begin{array}{c}
2048 m^{5}+7680 m^{4}+12096 m^{3} \\
+10368 m^{2}+4374 m+729
\end{array}\right) \ln ^{4} K}{3 m^{4}}-\frac{(2 m+3) \Delta_{2}}{3}, \\
& \Delta_{4}=-\frac{3\left(\begin{array}{c}
2048 m^{5}+7680 m^{4}+12096 m^{3} \\
+8208 m^{2}+2430 m+243
\end{array}\right) \ln ^{6} K}{m^{6}}+\frac{6 \Delta_{2}}{m} \ln ^{2} K .
\end{aligned}
$$

Substituting (80) together with (13) and (81) into (45), we attain the straddled soliton

$$
\begin{aligned}
q(x, t)= & \left\{\frac{4 A\left(\frac{9 \chi^{3}(4 m+3)(8 m+3)(2 m+3)(10 m+3)(2 m+1)}{e_{2}}\right)^{\frac{1}{6}} \ln K}{m\left[4 A^{2} \exp _{K}[2(x-c t)]+\chi \exp _{K}[-2(x-c t)]\right.}\right\}^{\frac{3}{2 m}} \\
& \times e^{i\left(-\kappa x+w t+\theta_{0}\right)} .
\end{aligned}
$$

With the help of $e_{2}>0$ and $\chi=4 A^{2}$, Equation (82) changes to the bright soliton

$$
\begin{aligned}
q(x, t)= & \left\{\frac{\ln K}{m}\left(\frac{9(8 m+3)(4 m+3)(10 m+3)(2 m+3)(2 m+1)}{e_{2}}\right)^{\frac{1}{6}}\right. \\
& \times e^{i\left(-\kappa x+w t+\theta_{0}\right)} .
\end{aligned}
$$


By the aid of $e_{2}<0$ and $\chi=-4 A^{2}$, Equation (82) collapses to the singular soliton

$$
\begin{aligned}
q(x, t)= & \left\{\frac{\ln K}{m}\left(-\frac{9(8 m+3)(4 m+3)(10 m+3)(2 m+3)(2 m+1)}{e_{2}}\right)^{\frac{1}{6}}\right. \\
\times \operatorname{csch}[2 \ln K(x-c t)] & \times e^{i\left(-\kappa x+w t+\theta_{0}\right)} .
\end{aligned}
$$

\section{Polynomial Law}

The polynomial law of nonlinearity is modeled as

$$
F\left(\phi^{2}\right)=e_{1} \phi^{2}+e_{2} \phi^{4}+e_{3} \phi^{6}, e_{3} \neq 0,
$$

where $e_{1}, e_{2}$ and $e_{3}$ are arbitrary constants. Therefore, Equation (1) simplifies to

$$
\begin{aligned}
& i q_{t}+i a_{1} q_{x}+a_{2} q_{x x}+i a_{3} q_{x x x}+a_{4} q_{x x x x}+i a_{5} q_{x x x x x}+a_{6} q_{x x x x x x} \\
+ & \left(e_{1}|q|^{2}+e_{2}|q|^{4}+e_{3}|q|^{6}\right) q=\alpha \frac{\left|q_{x}\right|^{2}}{q^{*}}+\frac{\beta}{4|q|^{2} q^{*}}\left\{2|q|^{2}\left(|q|^{2}\right)_{x x}-\left[\left(|q|^{2}\right)_{x}\right]^{2}\right\} \\
+ & \gamma q+i\left[\lambda\left(|q|^{2 m} q\right)_{x}+\mu\left(|q|^{2 m}\right)_{x} q+v|q|^{2 m} q_{x}\right],
\end{aligned}
$$

while Equation (9) collapses to

$$
\begin{aligned}
& \phi \phi^{(6)}+\Delta_{1} \phi \phi^{(4)}+\Delta_{2} \phi \phi^{\prime \prime}+\Delta_{3}\left(\phi^{\prime}\right)^{2}+\Delta_{4} \phi^{2} \\
+ & \Delta_{5} \phi^{2 m+2}+e_{1} \phi^{4}+e_{2} \phi^{6}+e_{3} \phi^{8}=0 .
\end{aligned}
$$

Setting $m=1$, Equation (87) transforms to

$$
\phi \phi^{(6)}+\Delta_{1} \phi \phi^{(4)}+\Delta_{2} \phi \phi^{\prime \prime}+\Delta_{3}\left(\phi^{\prime}\right)^{2}+\Delta_{4} \phi^{2}+\left(\Delta_{5}+e_{1}\right) \phi^{4}+e_{2} \phi^{6}+e_{3} \phi^{8}=0 .
$$

Balancing $\phi \phi^{(6)}$ and $\phi^{8}$ gives

$$
2 N+6 p=8 N \Longrightarrow N=p .
$$

Case 1: By virtue of $p=1$, Equation (88) holds the solution (39). Putting (39) along with (12) into (88) causes to the coefficients

$$
\sigma_{0}=0, \sigma_{1}=\left(\frac{720 \chi^{3}}{e_{3}}\right)^{\frac{1}{6}} \ln K,
$$

and the constraint conditions

$$
\begin{aligned}
& \Delta_{1}=-\frac{1}{24 \chi^{2}}\left[\frac{e_{2}}{e_{3}^{4}}\left(720 \chi^{3} e_{3}^{5}\right)^{2 / 3}+840 \chi^{2} \ln ^{2} K\right], \chi^{3} e_{3}>0, \\
& \Delta_{2}=\frac{1}{6 \chi^{2} e_{3}^{4}}\left[\begin{array}{c}
6 e_{3}^{2} \chi\left(90 \chi^{3} e_{3}^{5}\right)^{1 / 3}\left(e_{1}+\Delta_{5}\right)+10\left(\ln ^{2} K\right) e_{2}\left(90 \chi^{3} e_{3}^{5}\right)^{2 / 3} \\
-3 \chi^{2} e_{3}^{4}\left(\Delta_{3}-518 \ln ^{4} K\right)
\end{array}\right], \\
& \Delta_{4}=-\frac{3 \ln ^{2} K}{2 \chi^{2} e_{3}^{4}}\left[\begin{array}{c}
\frac{2}{3} e_{3}^{2} \chi\left(90 \chi^{3} e_{3}^{5}\right)^{1 / 3}\left(e_{1}+\Delta_{5}\right)+\left(\ln ^{2} K\right) e_{2}\left(90 \chi^{3} e_{3}^{5}\right)^{2 / 3} \\
+\frac{1}{3} \chi^{2} e_{3}^{4}\left(\Delta_{3}+450 \ln ^{4} K\right)
\end{array}\right] .
\end{aligned}
$$


Plugging (90) together with (13) and (91) into (39) gives rise to the straddled soliton

$$
q(x, t)=\left\{\frac{4 A\left(\frac{720 \chi^{3}}{e_{3}}\right)^{\frac{1}{6}} \ln K}{4 A^{2} \exp _{K}(x-c t)+\chi \exp _{K}(-(x-c t))}\right\} e^{i\left(-\kappa x+w t+\theta_{0}\right)} .
$$

When $e_{3}>0$ and $\chi=4 A^{2}$, Equation (92) becomes the bright soliton

$$
q(x, t)=\left\{\left(\frac{720}{e_{3}}\right)^{\frac{1}{6}} \ln K \operatorname{sech}[(x-c t) \ln K]\right\} e^{i\left(-\kappa x+w t+\theta_{0}\right)} .
$$

If $e_{3}<0$ and $\chi=-4 A^{2}$, Equation (92) turns into the singular soliton

$$
q(x, t)=\left\{\left(-\frac{720}{e_{3}}\right)^{\frac{1}{6}} \ln K \operatorname{csch}[(x-c t) \ln K]\right\} e^{i\left(-\kappa x+w t+\theta_{0}\right)} .
$$

Case 2: When $p=2$, Equation (88) permits the solution (45). Substituting (45) along with (12) into (88) yields the coefficients

$$
\sigma_{0}=\sigma_{1}=0, \sigma_{2}=2\left(\frac{720 \chi^{3}}{e_{3}}\right)^{\frac{1}{6}} \ln K,
$$

and the parameter conditions

$$
\begin{aligned}
& \Delta_{1}=-\frac{1}{24 \chi^{2}}\left[\frac{e_{2}}{e_{3}^{4}}\left(720 \chi^{3} e_{3}^{5}\right)^{2 / 3}+3360 \chi^{2} \ln ^{2} K\right], \chi^{3} e_{3}>0 \\
& \Delta_{2}=\frac{1}{6 \chi^{2} e_{3}^{4}}\left[\begin{array}{c}
6 e_{3}^{2} \chi\left(90 \chi^{3} e_{3}^{5}\right)^{1 / 3}\left(e_{1}+\Delta_{5}\right)+40\left(\ln ^{2} K\right) e_{2}\left(90 \chi^{3} e_{3}^{5}\right)^{2 / 3} \\
-3 \chi^{2} e_{3}^{4}\left(\Delta_{3}-8288 \ln ^{4} K\right)
\end{array}\right] \\
& \Delta_{4}=-\frac{6 \ln ^{2} K}{\chi^{2} e_{3}^{4}}\left[\begin{array}{c}
\frac{2}{3} e_{3}^{2} \chi\left(90 \chi^{3} e_{3}^{5}\right)^{1 / 3}\left(e_{1}+\Delta_{5}\right)+4\left(\ln ^{2} K\right) e_{2}\left(90 \chi^{3} e_{3}^{5}\right)^{2 / 3} \\
+\frac{1}{3} \chi^{2} e_{3}^{4}\left(\Delta_{3}+7200 \ln ^{4} K\right)
\end{array}\right] .
\end{aligned}
$$

Inserting (95) together with (13) and (96) into (45) gives the straddled soliton

$$
q(x, t)=\left\{\frac{8 A\left(\frac{720 \chi^{3}}{e_{3}}\right)^{\frac{1}{6}} \ln K}{4 A^{2} \exp _{K}[2(x-c t)]+\chi \exp _{K}[-2(x-c t)]}\right\} e^{i\left(-\kappa x+w t+\theta_{0}\right)} .
$$

Setting $e_{2}>0$ and $\chi=4 A^{2}$, Equation (97) transforms to the bright soliton

$$
q(x, t)=\left\{2 \ln K\left(\frac{720}{e_{3}}\right)^{\frac{1}{6}} \operatorname{sech}[2 \ln K(x-c t)]\right\} e^{i\left(-\kappa x+w t+\theta_{0}\right)} .
$$

If $e_{2}<0$ and $\chi=-4 A^{2}$, Equation (97) simplifies to the singular soliton

$$
q(x, t)=\left\{2 \ln K\left(-\frac{720}{e_{3}}\right)^{\frac{1}{6}} \operatorname{csch}[2 \ln K(x-c t)]\right\} e^{i\left(-\kappa x+w t+\theta_{0}\right)} .
$$




\section{Triple Power Law}

The triple power law of nonlinearity is structured as

$$
F\left(\phi^{2}\right)=e_{1} \phi^{2 n}+e_{2} \phi^{4 n}+e_{3} \phi^{6 n}, e_{3} \neq 0,
$$

where $e_{1}, e_{2}$ and $e_{3}$ are arbitrary constants. Thus, Equation (1) turns into

$$
\begin{aligned}
& i q_{t}+i a_{1} q_{x}+a_{2} q_{x x}+i a_{3} q_{x x x}+a_{4} q_{x x x x}+i a_{5} q_{x x x x x}+a_{6} q_{x x x x x x} \\
+ & \left(e_{1}|q|^{2 n}+e_{2}|q|^{4 n}+e_{3}|q|^{6 n}\right) q=\alpha \frac{\left|q_{x}\right|^{2}}{q^{*}}+\frac{\beta}{4|q|^{2} q^{*}}\left\{2|q|^{2}\left(|q|^{2}\right)_{x x}-\left[\left(|q|^{2}\right)_{x}\right]^{2}\right\} \\
+ & \gamma q+i\left[\lambda\left(|q|^{2 m} q\right)_{x}+\mu\left(|q|^{2 m}\right)_{x} q+v|q|^{2 m} q_{x}\right]
\end{aligned}
$$

while Equation (9) reduces to

$$
\begin{gathered}
\phi \phi^{(6)}+\Delta_{1} \phi \phi^{(4)}+\Delta_{2} \phi \phi^{\prime \prime}+\Delta_{3}\left(\phi^{\prime}\right)^{2}+\Delta_{4} \phi^{2} \\
+\quad \Delta_{5} \phi^{2 m+2}+e_{1} \phi^{2 n+2}+e_{2} \phi^{4 n+2}+e_{3} \phi^{6 n+2}=0 .
\end{gathered}
$$

Setting $n=m$, Equation (102) changes to

$$
\begin{array}{cc} 
& \phi \phi^{(6)}+\Delta_{1} \phi \phi^{(4)}+\Delta_{2} \phi \phi^{\prime \prime}+\Delta_{3}\left(\phi^{\prime}\right)^{2}+\Delta_{4} \phi^{2} \\
+\quad & \left(\Delta_{5}+e_{1}\right) \phi^{2 m+2}+e_{2} \phi^{4 m+2}+e_{3} \phi^{6 m+2}=0 .
\end{array}
$$

Balancing $\phi \phi^{(6)}$ and $\phi^{6 m+2}$ yields $N=\frac{1}{m}$. Setting

$$
\phi(\xi)=[U(\xi)]^{\frac{1}{m}},
$$

Equation (103) becomes

$$
\begin{aligned}
& -(2 m-1)(m-1)(4 m-1)(3 m-1)(5 m-1)\left[U^{\prime}\right]^{6} \\
+ & 15 m(2 m-1)(m-1)(4 m-1)(3 m-1) U U^{\prime \prime} U^{\prime \prime} \\
- & \Delta_{1} m^{2}(2 m-1)(m-1)(3 m-1) U^{2} U^{\prime 4}-20 m^{2}(2 m-1)(m-1)(3 m-1) U^{2} U^{\prime} U^{\prime \prime \prime} \\
- & 45 m^{2}(2 m-1)(m-1)(3 m-1) U^{2} U^{\prime \prime 2} U^{\prime 2}+6 \Delta_{1} m^{3}(2 m-1)(m-1) U^{3} U^{\prime \prime} U^{\prime 2} \\
+ & 15 m^{3}(2 m-1)(m-1) U^{3} U^{(4)} U^{\prime 2}-m^{4}\left[(m-1) \Delta_{2}-\Delta_{3}\right] U^{4} U^{\prime 2} \\
+ & 60 m^{3}(2 m-1)(m-1) U^{3} U^{\prime \prime \prime} U^{\prime \prime} U^{\prime}-4 \Delta_{1}(m-1) m^{4} U^{4} U^{\prime \prime \prime} U^{\prime} \\
- & 6(m-1) m^{4} U^{4} U^{(5)} U^{\prime}+15 m^{3}(m-1)(2 m-1) U^{3}\left(U^{\prime \prime}\right)^{3}-3 \Delta_{1} m^{4}(m-1) U^{4}\left(U^{\prime \prime}\right)^{2} \\
- & 15 m^{4}(m-1) U^{4} U^{(4)} U^{\prime \prime}+m^{5} \Delta_{2} U^{5} U^{\prime \prime}-10 m^{4}(m-1) U^{4}\left(U^{\prime \prime \prime}\right)^{2}+m^{5} \Delta_{1} U^{5} U^{(4)} \\
+ & m^{5} U^{5} U^{(6)}+m^{6} e_{3} U^{12}+m^{6} e_{2} U^{10}+m^{6}\left(e_{1}+\Delta_{5}\right) U^{8}+m^{6} \Delta_{4} U^{6}=0 .
\end{aligned}
$$

Balancing $U^{5} U^{(6)}$ and $U^{12}$ gives

$$
6 N+6 p=12 \Longrightarrow N=p .
$$

Case 1: When $p=1$, Equation (105) admits the solution (39). Inserting (39) along with (12) into (105) leads to the coefficients

$$
\sigma_{0}=0, \sigma_{1}=\frac{\ln K}{m}\left(\frac{(2 m+1)(m+1)(4 m+1)(3 m+1)(5 m+1) \chi^{3}}{e_{3}}\right)^{\frac{1}{6}},
$$


and the parameter constraints

$$
\begin{aligned}
& \Delta_{1}=-\frac{1}{m^{2} e_{3}^{4}(2 m+1)(m+1)(3 m+1) \chi^{2}} \\
& \times\left\{m^{2} e_{2}\left[\chi^{3} e_{3}^{5}(2 m+1)(m+1)(4 m+1)(3 m+1)(5 m+1)\right]^{2 / 3}\right. \\
& \left.+480 e_{3}^{4} \chi^{2}(m+1)\left(m+\frac{1}{3}\right)\left(m^{2}+\frac{3}{5} m+\frac{3}{20}\right)\left(m+\frac{1}{2}\right) \ln ^{2} K\right\}, \\
& \Delta_{2}=\frac{1}{m^{4}(2 m+1)(m+1)(3 m+1) \chi^{2} e_{3}^{4}}\left\{-(3 m+1)(2 m+1) m^{4} \Delta_{3} e_{3}^{4} \chi^{2}\right. \\
& +4 m^{2}\left(m^{2}+m+\frac{1}{2}\right) e_{2}\left(\ln ^{2} K\right)\left[(2 m+1)(m+1)(4 m+1)(3 m+1)(5 m+1) \chi^{3} e_{3}^{5}\right]^{2 / 3} \\
& +m^{4}(3 m+1)(2 m+1) e_{3}^{2} \chi\left(e_{1}+\Delta_{5}\right)\left[(2 m+1)(m+1)(4 m+1)(3 m+1)(5 m+1) \chi^{3} e_{3}^{5}\right]^{1 / 3} \\
& \left.+(3 m+1)(2 m+1)(m+1)\left(64 m^{4}+96 m^{3}+72 m^{2}+24 m+3\right) e_{3}^{4} \chi^{2} \ln ^{4} K\right\}, \\
& \Delta_{4}=-\frac{\ln ^{2} K}{m^{6}(m+1)(3 m+1) \chi^{2} e_{3}^{4}} \\
& \times\left\{m^{2}(2 m+1) \ln ^{2} K e_{2}\left[\chi^{3} e_{3}^{5}(2 m+1)(m+1)(4 m+1)(3 m+1)(5 m+1)\right]^{2 / 3}\right. \\
& +m^{4}(3 m+1)\left(e_{1}+\Delta_{5}\right) e_{3}^{2} \chi\left[\chi^{3} e_{3}^{5}(2 m+1)(m+1)(4 m+1)(3 m+1)(5 m+1)\right]^{1 / 3} \\
& \left.+(3 m+1) e_{3}^{4} \chi^{2}\left[\Delta_{3} m^{5}+(m+1)(4 m+1)^{2}(2 m+1)^{2} \ln ^{4} K\right]\right\}, \chi^{3} e_{3}>0 .
\end{aligned}
$$

Substituting (107) together with (13) and (108) into (39), we arrive the straddled soliton

$$
\begin{aligned}
q(x, t)= & \left\{\frac{4 A\left(\frac{(2 m+1)(m+1)(4 m+1)(3 m+1)(5 m+1) \chi^{3}}{e_{3}}\right)^{\frac{1}{6}} \ln K}{m\left[4 A^{2} \exp _{K}(x-c t)+\chi \exp _{K}(-(x-c t))\right]}\right\}^{\frac{1}{m}} \\
& \times e^{i\left(-\kappa x+w t+\theta_{0}\right)} .
\end{aligned}
$$

If $e_{3}>0$ and $\chi=4 A^{2}$, Equation (109) reduces to the bright soliton

$$
\left.\begin{array}{rl}
q(x, t)= & \left\{\frac{\ln K}{m}\left(\frac{(2 m+1)(m+1)(4 m+1)(3 m+1)(5 m+1)}{e_{3}}\right)^{\frac{1}{6}}\right. \\
\times \operatorname{sech}[(x-c t) \ln K]
\end{array}\right\}
$$

When $e_{3}<0$ and $\chi=-4 A^{2}$, Equation (109) changes to the singular soliton

$$
\left.\begin{array}{rl}
q(x, t)= & \left\{\frac{\ln K}{m}\left(-\frac{(2 m+1)(m+1)(4 m+1)(3 m+1)(5 m+1)}{e_{3}}\right)^{\frac{1}{6}}\right. \\
\times \operatorname{csch}[(x-c t) \ln K]
\end{array}\right\}^{\frac{1}{m}}
$$


Case 2: If $p=2$, Equation (105) presumes the solution (45). Plugging (45) along with (12) into (105) gives rise to the coefficients

$$
\sigma_{0}=\sigma_{1}=0, \sigma_{2}=\frac{2 \ln K}{m}\left(\frac{(2 m+1)(m+1)(4 m+1)(3 m+1)(5 m+1) \chi^{3}}{e_{3}}\right)^{\frac{1}{6}},
$$

and the parameter conditions

$$
\begin{aligned}
& \Delta_{1}=-\frac{1}{m^{2}(m+1)(2 m+1)(3 m+1) \chi^{2}} \\
& \times\left\{\frac{m^{2} e_{2}}{e_{3}^{4}}\left[\chi^{3} e_{3}^{5}(2 m+1)(m+1)(4 m+1)(3 m+1)(5 m+1)\right]^{2 / 3}\right. \\
& \left.+120 \chi^{2}(m+1)\left(m+\frac{1}{3}\right)\left(m^{2}+\frac{3}{5} m+\frac{3}{20}\right)\left(m+\frac{1}{2}\right) \ln ^{2} K\right\},
\end{aligned}
$$

$$
\begin{aligned}
& \Delta_{2}=\frac{1}{m^{4}(2 m+1)(m+1)(3 m+1) \chi^{2} e_{3}^{4}}\left\{-(3 m+1)(2 m+1) m^{4} \Delta_{3} e_{3}^{4} \chi^{2}\right. \\
+ & 16 m^{2}\left(m^{2}+m+\frac{1}{2}\right) e_{2} \ln ^{2} K\left[(2 m+1)(m+1)(4 m+1)(3 m+1)(5 m+1) \chi^{3} e_{3}^{5}\right]^{2 / 3} \\
+ & m^{4}(3 m+1)(2 m+1) e_{3}^{2} \chi\left(e_{1}+\Delta_{5}\right)\left[(2 m+1)(m+1)(4 m+1)(3 m+1)(5 m+1) \chi^{3} e_{3}^{5}\right]^{1 / 3} \\
+ & \left.(2 m+1)(3 m+1)\left(1024 m^{4}+1536 m^{3}+1152 m^{2}+384 m+48\right)(m+1) e_{3}^{4} \chi^{2} \ln ^{4} K\right\}, \\
& \Delta_{4}=-\frac{12 \ln ^{2} K}{m^{6}(m+1)(3 m+1) \chi^{2} e_{3}^{4}} \\
+ & \left\{\frac{4}{3} m^{2}(2 m+1) \ln ^{2} K e_{2}\left[\chi^{3} e_{3}^{5}(2 m+1)(m+1)(4 m+1)(3 m+1)(5 m+1)\right]^{2 / 3}\right. \\
+ & \frac{(3 m+1)\left(e_{1}+\Delta_{5}\right) e_{3}^{2} \chi\left[\chi^{3} e_{3}^{5}(2 m+1)(m+1)(4 m+1)(3 m+1)(5 m+1) e_{3}^{4} \chi^{2}\right.}{3}\left\{\left[\Delta_{3} m^{5}+16(m+1)(4 m+1)^{2}(2 m+1)^{2} \ln ^{4} K\right]\right\}, \chi^{3} e_{3}>0 .
\end{aligned}
$$

Inserting (112) together with (13) and (113) into (45) causes to the straddled soliton

$$
\begin{aligned}
q(x, t)= & \left\{\frac{8 A\left(\frac{(2 m+1)(m+1)(4 m+1)(3 m+1)(5 m+1) \chi^{3}}{e_{3}}\right)^{\frac{1}{6}} \ln K}{m\left\{\chi \exp _{K}(-2(x-c t))+4 A^{2} \exp _{K}(2(x-c t))\right\}}\right\}^{\frac{1}{m}} \\
& \times e^{i\left(-\kappa x+w t+\theta_{0}\right)} .
\end{aligned}
$$

Setting $e_{3}>0$ and $\chi=4 A^{2}$, Equation (114) simplifies to the bright soliton

$$
\begin{aligned}
q(x, t)= & \left\{\begin{array}{c}
\frac{2 \ln K}{m}\left(\frac{(2 m+1)(m+1)(4 m+1)(3 m+1)(5 m+1)}{e_{3}}\right)^{\frac{1}{6}} \\
\times \operatorname{sech}[2 \ln K(x-c t)]
\end{array}\right\}^{\frac{1}{m}} \\
& \times e^{i\left(-\kappa x+w t+\theta_{0}\right)} .
\end{aligned}
$$


When $e_{3}<0$ and $\chi=-4 A^{2}$, Equation (114) transforms to the singular soliton

$$
\left.\begin{array}{rl}
q(x, t)= & \left\{\frac{2 \ln K}{m}\left(-\frac{(2 m+1)(m+1)(4 m+1)(3 m+1)(5 m+1)}{e_{3}}\right)^{\frac{1}{6}}\right. \\
\times \operatorname{csch}[2 \ln K(x-c t)]
\end{array}\right\}^{\frac{1}{m}}
$$

\section{Conclusions}

This paper reports HD solitons with the perturbed CGLE having six forms of nonlinear refractive index structures. The perturbation terms are all of Hamiltonian type and are with maximum intensity. The integration scheme is the enhanced Kudryashov approach that is the extended and generalized version of the pre-existing Kudryashov scheme. Thus, straddled, bright and singular soliton solutions emerge from the scheme for each of these six nonlinear forms that are exhibited with their respective parameter constraints. Likewise, one can obtain a large number of HD solitons of the model equations by taking a different selection to the parameters $p$ and $N$.

There is an abundance of results that have been retrieved on the perturbed CGLE $[16,28,29]$ where Hamiltonian type perturbation terms are studied with maximum intensity. A spectrum of cubic-quartic optical solitons for the perturbed CGLE having a variety of six forms of nonlinear refractive index structures are derived by eight powerful and prolific integration structures [28]. Additionally, conservation laws for pure-cubic optical solitons with the perturbed CGLE having eleven forms of nonlinear refractive index structures are derived with the implementation of Lie symmetry analysis [29]. Lastly, pure-cubic optical solitons with the perturbed CGLE having a dozen nonlinear refractive index structures are recovered by two integration schemes [16]. However, compared with $[16,28,29]$ that secure pure-cubic or cubic-quartic optical solitons with the model equation, HD solitons with the perturbed CGLE are given in the current paper for the very first time. The results of this work are with unprecendented novelty and thus carry tremendous value in further future development of the concept of HD solitons with CGLE and/or NLSE. The results are indeed promising.

Later, conservation laws will be reported. The variational principle would lead to the evolution of the soliton parameters in presence and/or absence of perturbative effects. Once these fundamental results are in place, one can move further along with the development of quasi-particle theory to suppress intra-channel collision of optical solitons. The quasistationarity will also be addressed to recover soliton solutions in presence of perturbation terms, be it Hamiltonian or non-Hamiltonian. These are just a few droplets of a wide and deep ocean!!

Author Contributions: Conceptualization, E.M.E.Z. and K.A.G.; methodology, M.E.-H.; software, A.B.; writing—original draft preparation, Y.Y.; writing—review and editing, H.M.A. All authors have read and agreed to the published version of the manuscript.

Funding: This research received no external funding.

Institutional Review Board Statement: Not applicable.

Informed Consent Statement: Not applicable.

Data Availability Statement: All data generated or analyzed during this study are included in this manuscript.

Acknowledgments: The authors thank the anonymous referees whose comments helped to improve the paper.

Conflicts of Interest: The authors declare no conflict of interest. 


\section{References}

1. Kudryashov, N.A. Periodic and solitary waves in optical fiber Bragg gratings with dispersive reflectivity. Chin. J. Phys. 2020, 66, 401-405. [CrossRef]

2. Kudryashov, N.A. The generalized Duffing oscillator. Commun. Nonlinear Sci. Numer. Simul. 2021, 93, 105526. [CrossRef]

3. Kudryashov, N.A. Model of propagation pulses in an optical fiber with a new law of refractive indices. Optik 2021, $248,168160$. [CrossRef]

4. Kudryashov, N.A. Method for finding highly dispersive optical solitons of nonlinear differential equations. Optik 2020, 206, 163550. [CrossRef]

5. Kudryashov, N.A. Highly dispersive solitary wave solutions of perturbed nonlinear Schrödinger equations. Appl. Math. Comput. 2020, 371, 124972. [CrossRef]

6. Kudryashov, N.A. Highly dispersive optical solitons of an equation with arbitrary refractive index. Regul. Chaotic Dyn. 2020, 25, 537-543. [CrossRef]

7. Kudryashov, N.A. Highly dispersive optical solitons of the generalized nonlinear eighth-order Schrödinger equation. Optik 2020, 206, 164335. [CrossRef]

8. Kan, K.V.; Kudryashov, N.A. Solitary waves described by a high-order system in optical fiber Bragg gratings with arbitrary refractive index. Math. Methods Appl. Sci. 2021, in press. [CrossRef]

9. Kudryashov, N.A. Solitary wave solutions of hierarchy with non-local nonlinearity. Appl. Math. Lett. 2020, 103, 106155. [CrossRef]

10. Kudryashov, N.A. Mathematical model of propagation pulse in optical fiber with power nonlinearities. Optik 2020, $212,164750$. [CrossRef]

11. Kudryashov, N.A. Solitary waves of the non-local Schrödinger equation with arbitrary refractive index. Optik 2021, 231, 166443. [CrossRef]

12. Qiu, Y.; Malomed, B.A.; Mihalache, D.; Zhu, X.; Peng, J.; He, Y. Generation of stable multi-vortex clusters in a dissipative medium with anti-cubic nonlinearity. Phys. Lett. A 2019, 383, 2579-2583. [CrossRef]

13. Shwetanshumala, S. Temporal solitons of modified complex Ginzberg-Landau equation. Prog. Electromagn. Res. Lett. 2008, 3, 17-24. [CrossRef]

14. Wazwaz, A.M. Explicit and implicit solutions for the one-dimensional cubic and quintic complex Ginzburg-Landau equations. Appl. Math. Lett. 2006, 19, 1007-1012. [CrossRef]

15. Yan, Y.; Liu, W. Stable transmission of solitons in the complex cubic-quintic Ginzburg-Landau equation with nonlinear gain and higher-order effects. Appl. Math. Lett. 2019, 98, 171-176. [CrossRef]

16. Zayed, E.M.E.; Alngar, M.E.M.; Biswas, A.; Ekici, M.; Khan, S.; Alshomrani, A.S. Pure-cubic optical soliton perturbation with complex Ginzburg-Landau equation having a dozen nonlinear refractive index structures. J. Commun. Technol. Electron. 2021, 66, 481-544. [CrossRef]

17. Zhao, Y.; Xia, C.-Y.; Zeng, H.-B. Cascade replication of soliton solutions in the one-dimensional complex cubic-quintic GinzburgLandau equation. Phys. Lett. A 2020, 384, 126395. [CrossRef]

18. Huang, C.; Li, Z. New exact solutions of the fractional complex Ginzburg-Landau equation. Math. Probl. Eng. 2021, 2021, 6640086. [CrossRef]

19. Kudryashov, N.A. First integrals and general solution of the complex Ginzburg-Landau equation. Appl. Math. Comput. 2020, 386, 125407. [CrossRef]

20. Sakaguchi, H.; Malomed, B. Solitary pulses and periodic waves in the parametrically driven complex Ginzburg-Landau equation. J. Phys. Soc. Jpn. 2003, 72, 1360-1365.

[CrossRef]

21. Avci, M.; Süer, B. On a nonlocal problem involving a nonstandard nonhomogeneous differential operator. J. Elliptic Parabol. Equ. 2019, 5, 47-67. [CrossRef]

22. Ginzburg, V.; Landau, L. On the theory of superconductivity. Zh. Exper. Teor. Fiz. 1950, 20, 1064-1082.

23. Bethuel, F.; Brezis, H.; Hlein, F. Ginzburg-Landau Vortices, Birkhuser: Basel, Switzerland, 1994.

24. Comte, M.; Mironescu, P. Minimizing properties of arbitrary solutions to the Ginzburg-Landau equation. Proc. R. Soc. Edinb. Sect. A Math. 1999, 129, 1157-1169. [CrossRef]

25. Mironescu, P. On the stability of radial solutions of the Ginzburg-Landau equation. J. Func. Anal. 1995, 130, 334-344. [CrossRef]

26. Struwe, M. Une estimation asymptotique pour le modle de Ginzburg-Landau. Comptes Rendus Acad. Sci. Paris 1993, 317, 677-680.

27. Akram, G.; Mahak, N. Application of the first integral method for solving (1+1)-dimensional cubic-quintic complex GinzburgLandau equation. Optik 2018, 164, 210-217. [CrossRef]

28. Biswas, A.; Yildirim, Y.; Ekici, M.; Guggilla, P.; Khan, S.; Gonzalez-Gaxiola, O.; Alzahrani, A.K.; Belic, M.R. Cubic-quartic optical soliton pertubation with complex Ginzburg-Landau equation. J. Appl. Eng. Sci. 2021, 24, 937-1004.

29. Biswas, A.; Kara, A.H.; Sun, Y.; Zhou, Q.; Yildirim, Y.; Alshehri, H.M. Conservation laws for pure-cubic optical solitons with complex Ginzburg-Landau equation having several refractive index structures. Results Phys. 2021, 31, 104901. [CrossRef]

30. Mou, D.S.; Fang, J.J.; Fan, Y. Discrete localized excitations for discrete conformable fractional cubic-quintic Ginzburg-Landau model possessing the non-local quintic term. Optik 2021, 244, 167554. [CrossRef]

31. Naghshband, S.; Araghi, M.A.F. Solving generalized quintic complex Ginzburg-Landau equation by homotopy analysis method. Ain Shams Eng. J. 2018, 9, 607-613. [CrossRef] 